\title{
Direct imaging on the deformation and sintering of polymeric particles at the nanoscale by liquid-phase TEM
}

\author{
Chang Liu ${ }^{1}$, Zihao $\mathrm{Ou}^{2}$ and Qian $\mathrm{Chen}^{3}$ \\ ${ }^{1}$ University of Illinois at Urbana-Champaign, United States, ${ }^{2}$ Stanford, California, United States, \\ ${ }^{3}$ University of Illinois at Urbana-Champaign, Urbana, Illinois, United States
}

Polymer-based coatings are widely applied in modern industry and technology. While volatile organic solvents were used conventionally for the formation of polymer coatings, the world-wide environmental concerns they could bring about have been calling for replacement with water-based charge-stabilized colloidal suspension. In colloid-based coatings, during the drying process of the solvent, the colloidal particles fuse together and eventually form the coating, the mechanical and corrosion resistance properties of which sensitively depend on the internal morphology. However, the understanding on the pathway of the formation of a dense coating with specific morphology from individual colloids, which is critical to the final morphology, is largely missing, especially at the single-particle level. The colloidal suspension composed of polymeric colloids and multiple ionic groups can go through complex processes during sintering and coating formation. On one hand, the interplay among particle diffusion, particle interactions, interfacial tensions, and solvent evaporation introduce different categories of processes and stages in the sintering [1-3]. On the other hand, the corresponding concentrations and the environmental conditions of the components undergoing changes that can potentially make a difference in the final properties of coating [4]. Scanning electron microscopy, cryo electron microscopy, and optical microscopy have been used to study the microstructure of coatings as well as the dynamics of the colloids [5-7], less has been done on the connection of chain interpenetration, crystallinity with particle deformation, or on how chain the ionic groups reorganize at various stages of film formation. In comparison, liquid-phase transmission electron microscope (TEM) can provide direct information of the sintering process at proper electron dose rate [8-9], especially the dynamics at the nanoscale such as particle deformation and fusion.

Here, by using liquid-phase TEM, we managed to capture the sintering process of polystyrene (PS) spheres of 100 to $200 \mathrm{~nm}$ in size at real time and real space. The negatively charged PS spheres stay locally on the positively charged $\mathrm{SiN}_{x}$ membrane [10], making it easier for the observation of the deformation dynamics. Specifically, the PS spheres were dispersed in deionized water and loaded into a Hummingbird liquid holder with a spacer chip of $250 \mathrm{~nm}$ in thickness. The high cross-linking density endows the PS spheres

with generic TEM contrast as shown in Figure 1. Dose rates needs to be kept to be below $10 \mathrm{e}^{-} \AA^{-2} \mathrm{~s}^{-1}$; otherwise visible loss of PS materials into the surrounding solution due to beam-induced radiation damage was observed. As shown in Figure 1, as the solvent quality changed, the closely packed PS spheres were pushed against each other. Different from rigid inorganic nanoparticles, PS spheres are soft. Such physical contact and attraction between the spheres resulted in the flattening of PS boundaries. This observation suggests the uniqueness of soft polymeric colloids in their phase transitions - excluded volume repulsion in hard colloids is softened, allowing for deformation and even closer packing into a space-filling highquality coating film without interstices. More work on systematic studies of the beam effects and of the packing behaviors of PS spheres under changes of conditions such as drying or heating will be conducted to provide more insights on soft polymer colloids [11]. 

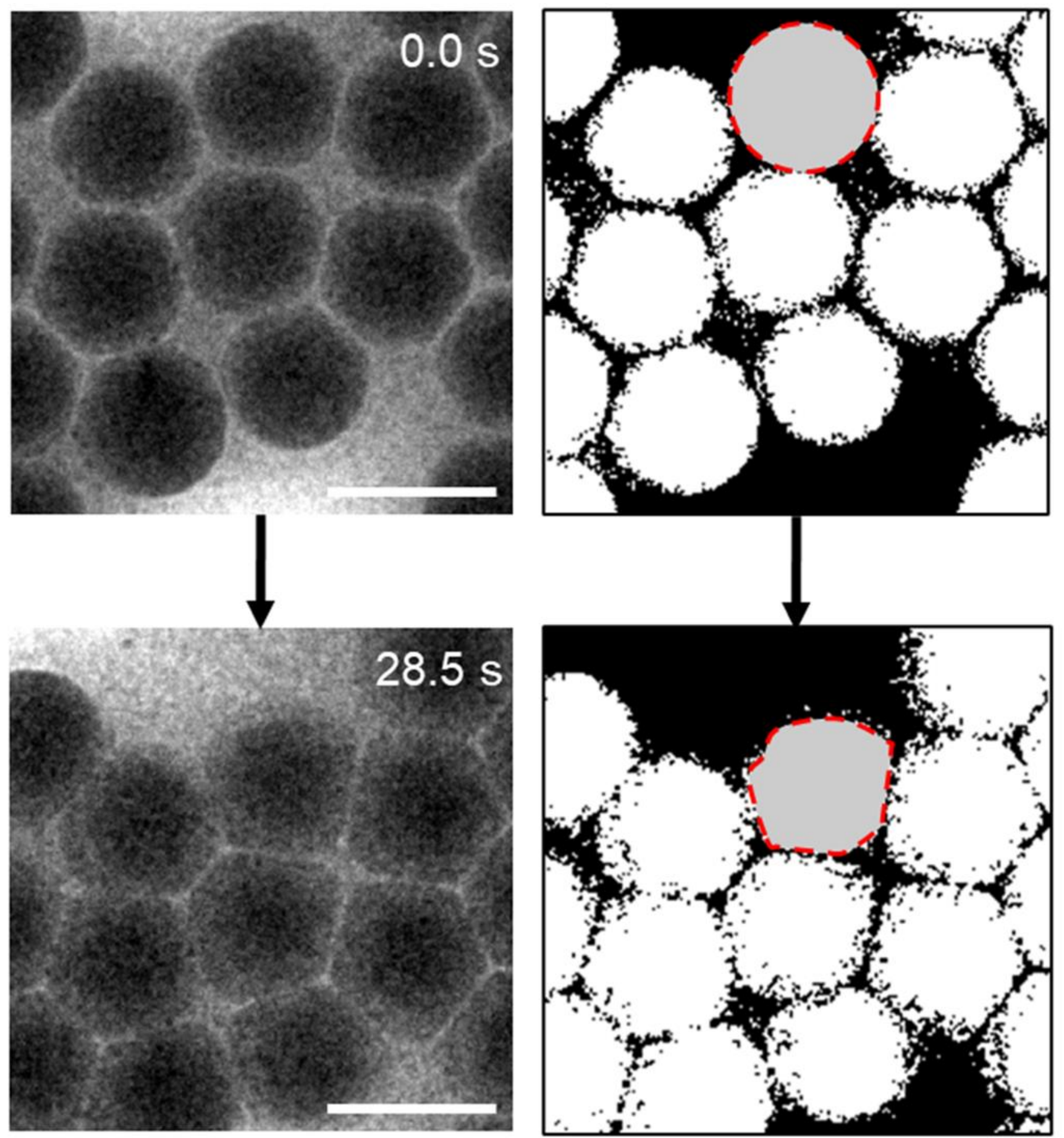

Figure 1. Snapshots of the deformation and sintering of PS spheres in a continuous liquid-phase TEM movie. Scale bars: $200 \mathrm{~nm}$.

\section{References}

[1] A F Routh, W B Russel. Industrial \& Engineering Chemistry Research 2001, 40, 4302-4308.

[2] Y Liu, et al., Macromolecules 2019, 52, 9536

[3] Carter, F. T.; Kowalczyk, R. M.; Millichamp, I.; Chainey, M.; Keddie, J. L., Langmuir 2014, 30,9672 
[4] B Delattre, et al., Journal of The Electrochemical Society 165 (2018), A388

[5] Ma, Y.; Davis, H. T.; Scriven, L. E., Microstructure development in drying latex coatings, Progress in Organic Coatings 2005, 52, 46-62.

[6] Yamamoto, S.; Nakazawa, S.; Sugisaki, K.; Sato, K.; Toyota, K.; Shiomi, D.; Takui, T., Adiabatic quantum computing with spin qubits hosted by molecules, Phys Chem Chem Phys 2015, 17, 2742-2749. [7] Casier, R.; Gauthier, M.; Duhamel, J., Using Pyrene Excimer Fluorescence To Probe Polymer Diffusion in Latex Films, Macromolecules 2017, 50, 1635-1644.

[8] B Luo, J W. Smith, Z Ou, Q Chen. Acc. Chem. Res. 50 (2017), p. 1125

[9] Z Ou, C Liu, L Yao. Accounts of Materials Research, 1 (1), pp 41-52 (2020)

[10] C Liu et al., Journal of the American Chemical Society, 142 (27), pp 11669-11673 (2020)

[11] The authors acknowledge funding from the National Science Foundation under Grant No. 1752517. 